\title{
Classification of texture using random box counting and binarization methods
}

\author{
Wijdan Jaber AL-kubaisy, Maha Mahmood \\ College of Computer Science and Information Technology, University of Anbar, Iraq
}

\begin{tabular}{l}
\hline \hline Article Info \\
\hline Article history: \\
Received Feb 26, 2020 \\
Revised Apr 30, 2020 \\
Accepted May 15, 2020 \\
\hline
\end{tabular}

\section{Keywords:}

Binarization method Box-counting method Fractal dimension, Texture recognition White blood cell

\begin{abstract}
The heterogeneous texture classifications with the complexity of structures provide variety of possibilities in image processing, as an example of the multifractal analysis features. The task of texture analysis is a highly significant field of study in the field of machine vision. Most of the real-life surfaces exhibit textures and an efficiently modelled vision system must have the ability to deal with this variety of surfaces. A considerable number of surfaces maintain a self-similarity quality as well as statistical roughness at different scales. Fractals could provide a great deal of advantages; also, they are popular in the process of modelling these properties in the tasks related to the field of image processing. With two distinct methods, this paper presents classification of texture using random box counting and binarization methods calculate the estimation measures of the fractal dimension BCM. There methods are the banalization and random selecting boxes. The classification of the white blood cells is presented in this paper based on the texture if it is normal or abnormal with the use of a number of various methods.
\end{abstract}

This is an open access article under the CC BY-SA license.

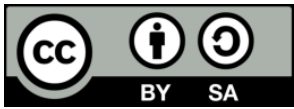

Corresponding Author:

Wijdan Jaber AL-kubaisy,

College of Computer Science and Information Technology,

University of Anbar,

Ramadi, Iraq.

Email:wijdan@computer-college.org

\section{INTRODUCTION}

There are number of choices that the multifractal analysis in image processing has provided. One good example of that, the classification of heterogeneous texture with a great complexity in the structure [1]. Visual textures are highly important in the interpretation of images as they include important image details, and as texture representations which pool the local image descriptors in order less approach have a significant effect on different applications [2]. At the other hand, great number of features is compromised mostly by the substantial problems which related to the data mining. However, some features might be inaccurate or not relevant which eventually effected the correctness of the classification algorithms. One of the most important and efficient methods is the texture analysis, it is implemented in the analysis and classification of images which show a redundancy in the pixels, and it's an important issue of the fields for image processing and computer vision [3]. The types of texture analysis are divided into 4 fundamental, which are: statistical, filter based, model based and structural. The idea of the fractal models was initially presented by Mandelbrot [4], it had indicated surface roughness. Fractal theory is one of many widely implemented approaches.

According to International Diabetes Federation (IDF) now people are having diabetes is about 387 million worldwide and it will be increased to 592 million by 2035. IDF declared that $52 \%$ of Indians do not know about diabetes that they are suffering with high blood sugar. In rural India around 34 million people affected with diabetes compared to urban Indians around 28 million people [5]. Many fractal approaches are used in different areas of science (like image processing) [6]. According to the surfaces, rough surfaces are 
the most common compared to smooth ones in nature, however, they exist in artificial environments too, like cloths, carpets, and microstructure textures existing on usually flat surfaces. Fractal geometry tools [7] typically model a rough surface. Thus, the fractal functions could be thought of as a sufficient option to represent surfaces of natural tissues that are chosen due to the short period importance of the image classification properties, as the surface fractal dimension has a complete correlation approximation to the toughness of surface [8]. Thereby, textures measure surface roughness. In grey level textures characterized by grey levels' distribution in an area, this is why, it is not possible to define textures precisely, but in a form of a pattern repeating of local change in the image intensity; Textures could be mathematically represented in a form of a function of things and patterns [9]. Other scholars depended on Bouligand-Minkowski descriptors; recursively decomposing initial images into equal portions and estimating the BouligandMinkowski descriptors' mean and deviation that are computed for each part, it obtains better outputs than texture conventional descriptors, like Gabor-wavelets, co-occurrence matrix and local binary patterns [10]. Other studies that implemented algorithms which provided more than one image slide based on an innovative method which produced multi thresholding and afterwards, obtains the characteristic of the fractal dimension utilizing the method of box counting every one of the slides [11].

\section{THE BLOOD}

Blood is a composition of liquid (named plasma) and solid cells, which are created in the bone marrow (a spongy, soft mass that resides in the bone center). In grown-ups, the marrow that has the maximal activity is in the spine bones (also referred to as the vertebrae), breast bone, bones of the pelvis, bones of the shoulders, rib bones, and the skull. Undeveloped blood cells in the marrow are known as the stem cells, residing in the bloodstream as well, but in smaller amounts, and are called peripheral blood stem cells, which could be abbreviated to (PBSCs) [12].

The stem cells are responsible for creating all human's blood cells. Haematopoiesis is the process which is responsible for the development of blood cells. Regarding the initial stage of the development of the blood cells, where stem cells are converted to blasts. The related cells for this line become oblasts of lymph and afterwards become lymphocytes that a kind of white blood cells, termed as leukocytes too. Those cells have a significant impact in resisting infections and getting rid of anomalous cells [13].

Myeloid cell line, those cells later become red blood cells (RBCs), several types of white blood cells, and platelets. The latter form clots in broken vessels in order to prevent bleeding. Whereas red blood cells deliver $\mathrm{O} 2$ to all tissues of the organism [14].There are a plenty of types of white blood cells. Every of those types have a certain part to play. Lymphocytes produce anti-bodies which have the task of fighting sickness. The main lymphocytes kinds are: 1) T cells are responsible for fighting infections, destroying anomalous cells and controlling the response of the immune system. 2) B cells are produce the anti-bodies that have the task of fighting bacteria, viruses and other unwanted material like fungus. 3) Natural killer (NK) cells have the responsibility of attacking foreign cells. Figure 1 shows the blood cell types [15].

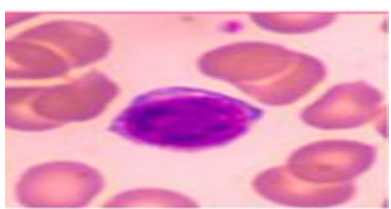

(a)

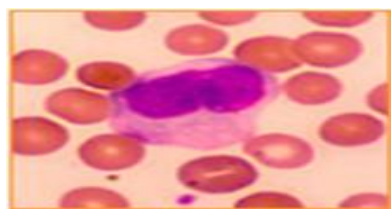

(b)



(c)

Figure 1. The blood cell types, (a) T-Lymphocyte, (b) B-Lymphocyte, (c) Natural killer-cell

\section{LEUKEMIA}

Leukemia is thought of as the condition that usually happens to children, however, it actually happens more in adults. It is more commonly met in male than females and also it is more commonly met in Caucasians than Africans. In fact, no one can control in order to avoid this condition happening to them. Leukemia is the cancer of blood cells which happens as a result of a rises number of white blood cells. Those cells push away the red blood cells and platelets that are necessary for the body in order to stay well. These excessive numbers of white blood cells do not properly operate, resulting in serious health issues. These leukemia cells cannot resist illnesses same as white blood cells do and due to the fact that there is too many of these cells, they start to affect the functioning of the major body parts. Therefore, the red blood cells count is not enough for providing and delivering $\mathrm{O}_{2}$, and the number of platelets is not sufficient to produce clots in 
the blood, nor a sufficient number of normal white blood cells to resist infections. Figure 2 describes the development of the cells [16].

In addition to infections, this could be resulting in issues like bruises, bleeding, and anemia. Leukemia have different types, this condition is classified on the basis of two considerations. First, according to how fast it develops and second, how fast it gets more serious. Based on the blood cell types involved (myeloid or lymphoid), these types are also classified as acute and chronic. Chronic leukemia the case in which is an amount of immature cells, and the rest can normally operate. That means it becomes serious, but at a slower pace. Acute leukemia is the situation in which most abnormal cells remain immature and cannot normally operate. In this case it could get more serious in a very short time.

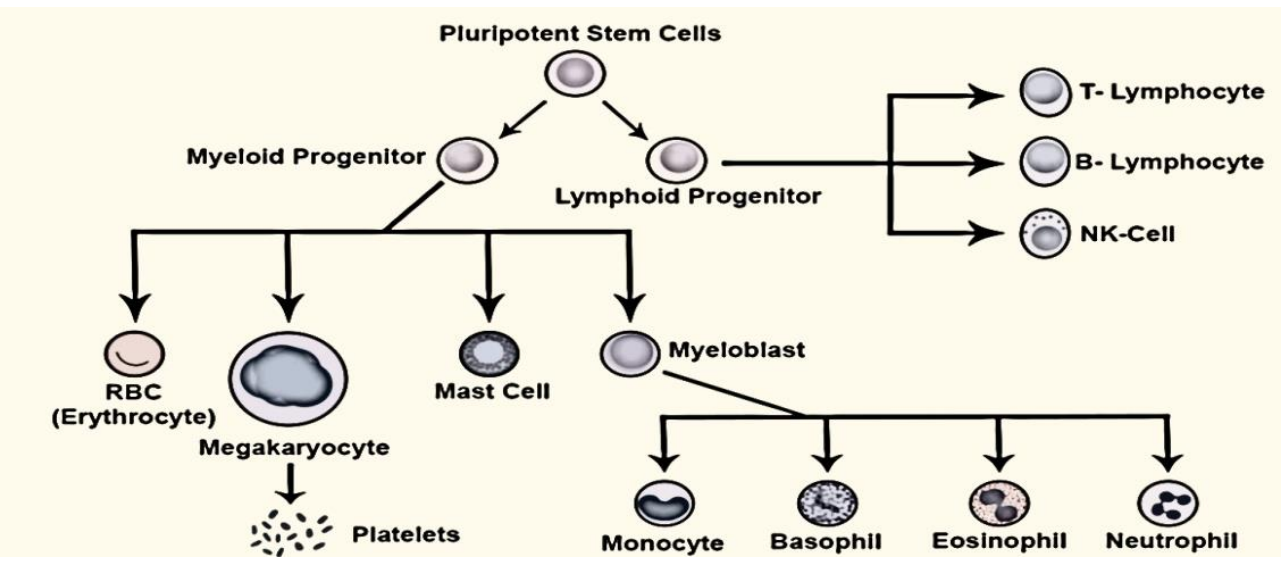

Figure 2. Cell development

\section{FRACTAL DIMENSION}

Fractal dimension (FD) is a very significant characteristic of fractal geometry, it is applied in many different applications, including image processing, analysis of images, classification of shapes, texture segmentation, and the identifying image characteristics like how rough or smooth it is. There are many different approaches for the estimation of fractal surface dimensions [17]. The commonly utilized method of the calculation of fractal dimensions is the grid dimension approach, also referred to as the box counting approach and a group of other enhanced methods like differential box counting and the approach of improved differential box counting are used for the estimation of greyscale images fractal dimension [18]. This characteristic is deployed in classifying and describing textures, analysis of shapes; it is responsible for providing an efficient base for invariance between different fractal objects. The multi-threshold algorithm is used to generate the binarization method which is in turn used to find the boxing counting method, and eventually calculate the FD. For each texture image, one or more than slide can be generated using the multithreading algorithm. On each slide pf these, a single FD will be applied to generate what it called a multifractal dimension. Moreover, for all the pixel of the textures image, there is a feature vector will be calculated as feature vector $M=\{m 1, m 2 \ldots, m n\}$. However, more experiments have been made to found the dimensions for specific objects. These experiments work well with lines, edge, spaces and so on [19].

\section{BOX COUNTING}

This approach is a rather common approach of fractal dimensions. The most significant reasons of its popularity are the comparatively easy calculation and mathematical estimation. For BCD estimation, according to (1) and (2), at first boxes A(s) number for whole block should be counted in order to resolve the overlapping issue which is related to the FD calculation for those blocks. The following equations are used to compute the fractal dimension [20]:

$$
\mathrm{H}=\frac{\left.\sum_{s=0}^{k-2} \log (A(s)) * \log 9 N(s)\right)}{\sum_{s=0}^{k-2} \log (A(s))^{2}}
$$

$\mathrm{FD}=2-\mathrm{H}$ 
$\mathrm{H}$ represents the slop, $\mathrm{A}(\mathrm{s})$ represents full boxes number of all blocks, $\mathrm{N}(\mathrm{s})$ define the number of boxes that are covering the design, the maximum size of a box is represented by $\mathrm{k}$, and $\mathrm{S}$ is the size of box. The curve FD value might be in the range between 1 (the topological dimension) and 2 (which is the plane's topological dimension).

\subsection{Binarization with mesh box counting method}

Due to the fact that the representation of binary images could be operated using high-speed logical operations (Boolean), it merely required a single bit to be assigned to every pixel ' 1 ' or ' 0 ' whereas found in a grey level. Typically, binary images could be resulted from abstracting the basic data from a grayscale image, such as the location of the object, its edges, or whether some characteristics of the image are present, which made the processing of binary images utilized for special tasks [20-21].

Thresholding is a procedure of converting a grey scale image to a black and white image via a proper threshold. The conventional thresholding utilizes a single threshold as a global one for every pixel, while adaptive thresholding changes the threshold over the image in a dynamic way. Taking under consideration that a grey level image $\mathrm{f}$ takes $\mathrm{K}$ potential grey-level values in the set of $\{0,1,2, ., \mathrm{K}-1\}$ [9]. Determining a threshold, $\mathrm{T}$ that has the value in the grey-scale range of $\{0,1,2, \ldots, \mathrm{K}-1\}$. The procedure of thresholding is simply done by comparing each the pixel value (f) with $\mathrm{T}$ [22]. According to this comparison, a binary decision ( 0 , if $\mathrm{f}$ is greater or equal to $\mathrm{T}$, otherwise 1$)$ is made, which determines the matching pixel value in an output binary image $(\mathrm{g})$.

There are a number of methods to calculate a threshold (histogram, traditional, actual, and others). One of the most widely utilized approaches for the calculation of the threshold is the histogram, it indicates the pixel count for every grey level volume in the grayscale for the image range. Peaks in a histogram point to increase redundant grey values in the image, typically comprising nearly uniform areas, whereas valleys point to the less redundant grey values. Empty regions indicate the absence of the pixels of those values in the image. In thresholding approaches, a threshold value must be determined. After the histogram calculation, the minimal and maximal values are specified (indicating low and high grey areas). This process might differ from one method to the other, such as in the traditional method the maximal and minimal values could be specified directly, the maximal and minimal values showed a component values lower and upper bound, in which the histogram elements summation beyond these bounds is below a predetermined value [23]. To compute the group of uniformly distributed threshold values; the calculation of intensity interval between the possible values of thresholds is:

$$
\text { Interval }=V_{1}-V_{2} \backslash n+1
$$

$\mathrm{n}$ denotes number of thresholds, v1 denotes peaks, v2 denotes valleys. Algorithm1 shows the steps of binarization via multi-thresholding by the use of the histogram method.

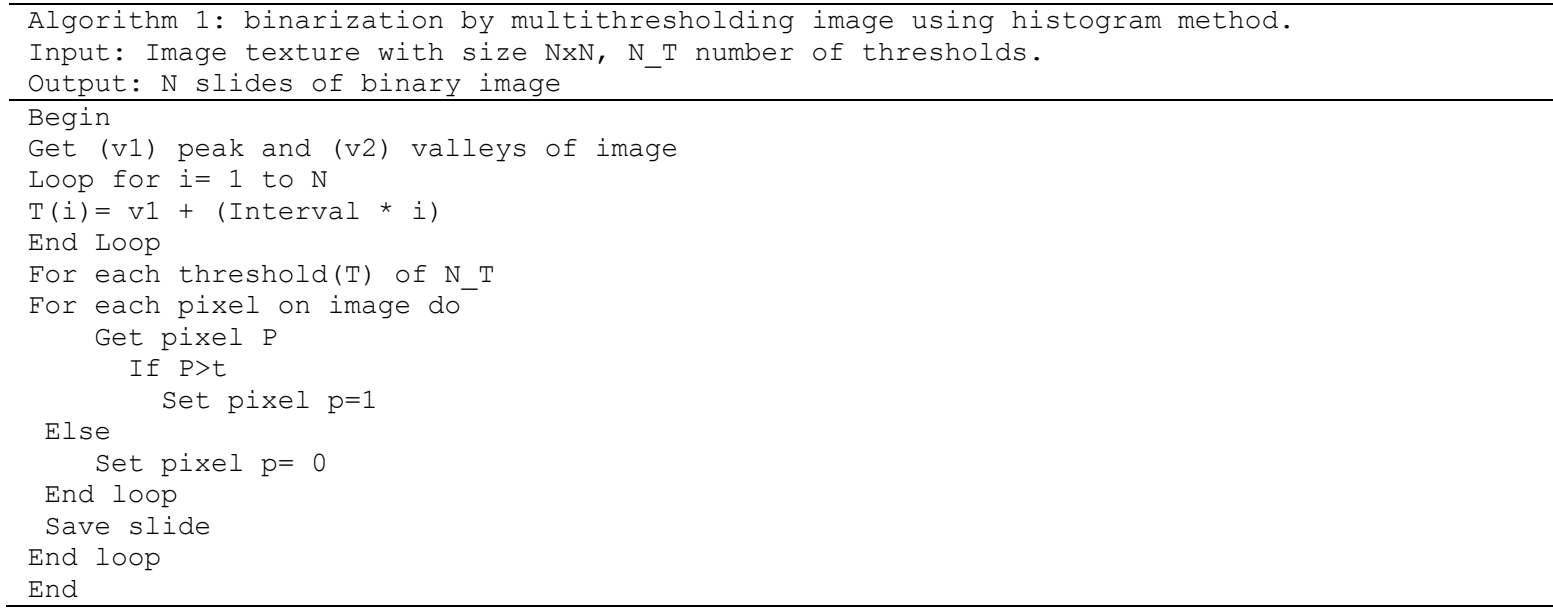

The sliding grey level image into several binary images by multi thresholding and binarization methods is illustrates in Figure 3. In Algorithm 2, the proposed method pseudo code is exhibited by stating the fundamental processes (binarization method) for the image texture. The pseudo code can be considered as the "skeleton" in the three-dimensional space with $\mathrm{x}$ and y coordinates representing the two dimensional position and $\mathrm{z}$ coordinate represents the shape of the object. Then, in each one of the binary images slide the size of boxes as: $(\mathrm{s}=2.32, \mathrm{~W} \bmod \mathrm{s}=0)$. 




Figure 3. Diagram of binarization and multi-thresholding methods

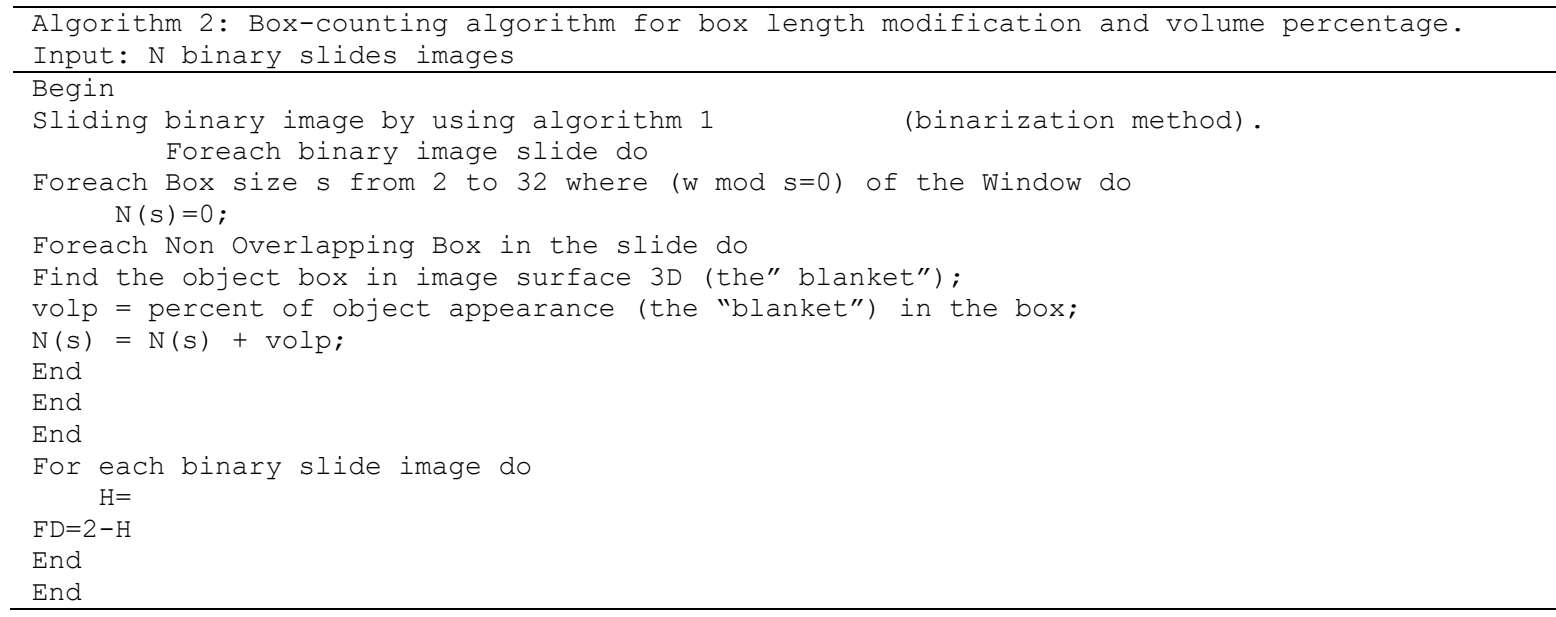

From each 3-D box, in order to calculate the "count" (N(s)) for each box size (s), distinct volume percentages are summed, $\mathrm{H}$ is the slop, $\mathrm{N}(\mathrm{s})$ represents the number of boxes that are covering the structure, $\mathrm{A}(\mathrm{s})$ is the boxes total number for all blocks, $\mathrm{k}$ denotes the maximal size of the box and $\mathrm{s}$ denotes the box size. A curve's FD might be in various values ranging from 1 (i.e. its topological dimension) to 2 (i.e. the topological dimension at which the plane can occupy) [24].

\subsection{Random box counting method}

Another approach of measuring the fractal dimensions by the approach of box counting was dependent on the arbitrary selection of boxes from image textures and measuring their fractal dimensions. Image texture is randomly split into smaller blocks that are of $\mathrm{M} \times \mathrm{M}$ size, where $\mathrm{M}$ must be of value bigger than 0 and smaller than the width and the height of the selected image texture of the size LH $\times \mathrm{LW}$. The initial pixel for each one of the blocks could be assigned in an arbitrary manner based on (4) and (5) [25]. 
$\mathrm{XS}=(\mathrm{LH}-\mathrm{M}) *$ rand

$\mathrm{YS}=(\mathrm{LW}-\mathrm{M}) *$ rand

YS, XS represent the initial pixel coordinates of every one of the blocks and "rand" is a function, which generates a random value in the range between $(1,0)$. Post the determination of the random blocks from the texture of the binary image. The threshold is utilized for converting grey rank to binary in this research it is 127 , the length of the block slide is altered a number of times, and 20 pixels every time, which starts from ten pixels to 90, finally it computes the fractal dimension. Algorithm 3 shows analyzing steps of computing blocking approach. The output from this algorithm statistical indicator of image texture topology. Figure 4 shows the approach of image texture BCM random selection stage and that includes selecting, calculating and extracting features [25].
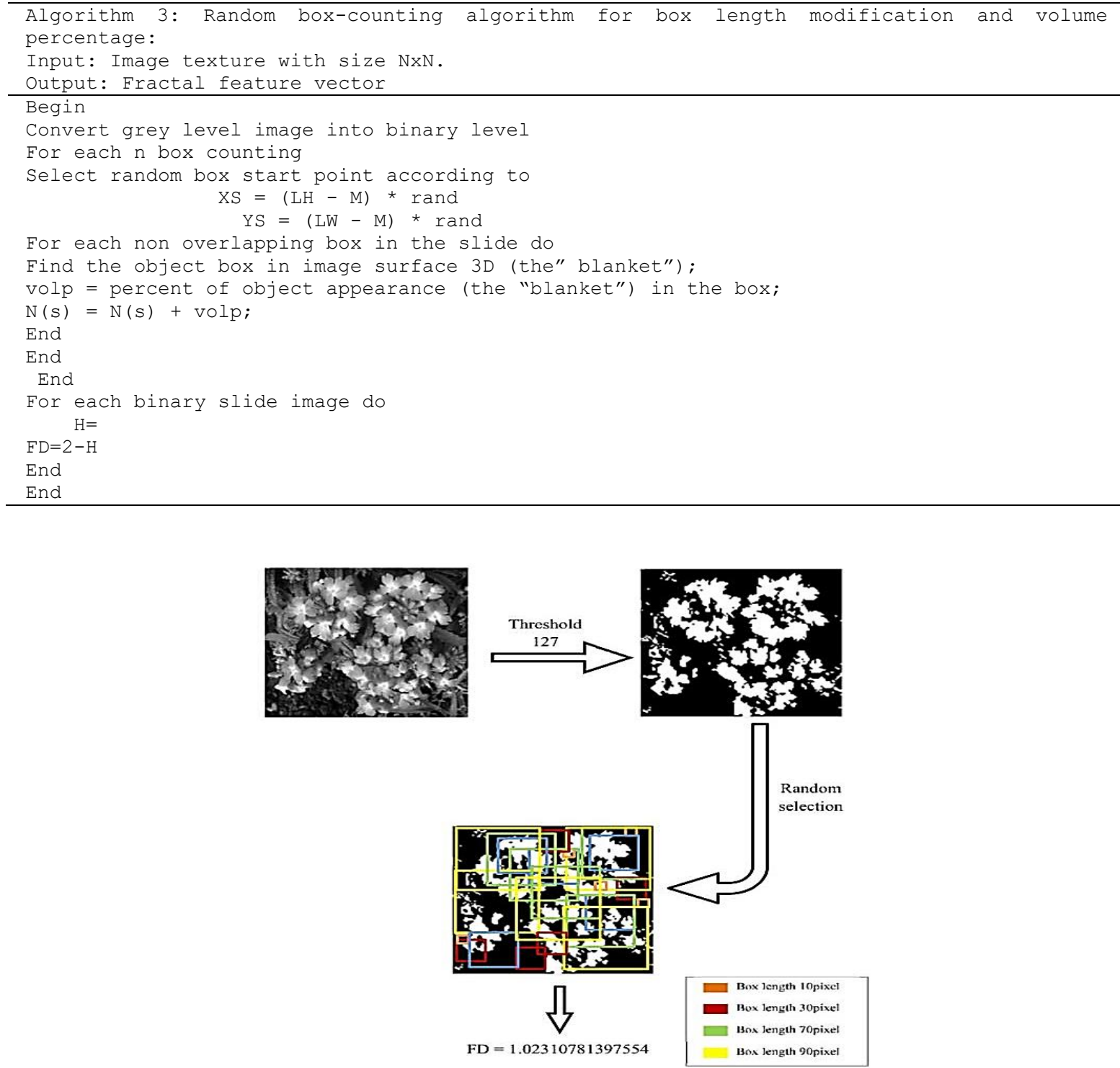

Figure 4. WBC the levels of the random selection technique for the image texture including select, calculate and extract feature

\section{RESULTS AND DISCUSSION}

Approach of fractal dimension binarization measure: this approach obtains the highest identification percentage compared to the utilized approaches, this method gets a highest recognition rate among used methods which was $96 \%$ for all of the classes. Figure 5 illustrates curve of recognition of the fractal dimension. 


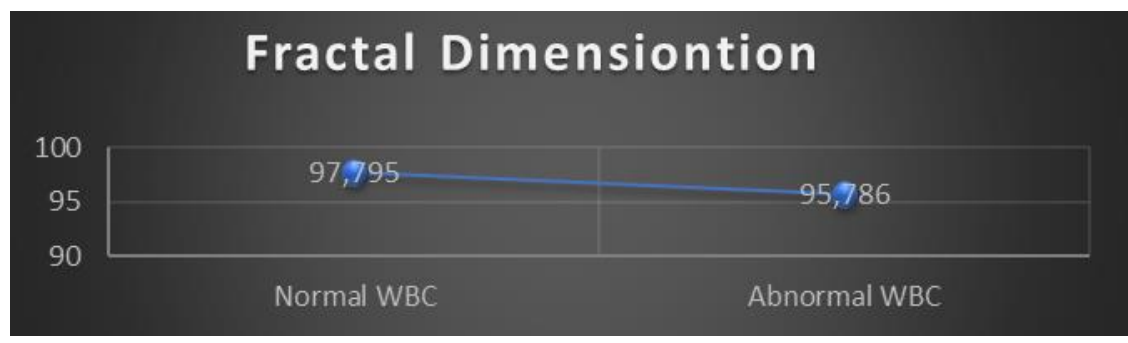

Figure 5. Recognition ratio of fractal dimension

In the approach of random box counting of fractal measures, the value of the fractal dimension of one image texture could differ when the procedure is done over on the texture of the image based on the chosen boxes. This is due to the randomly adopting selection of boxes from the texture of the image. This is why, the fractal dimension does not have a particular value (in contrast to the approach of binarization). Via using this approach in the discrimination system on the texture of the tested image it gets results of $80 \%$. The rate of recognition varies according to the feature container on boxes. In sliding technique from binarization method (fractal dimension), by trying from 8 to 12 slides for texture image, we found that sliding image to 10 slides get a better recognition rate. Random technique of fractal dimension measure does not need to make process on all texture image (as binarization method) it selects the boxes randomly, thus it has less processing and not fixed fractal dimension value than binarization method.

\section{CONCLUSION}

This paper presented the classification of texture using random box counting and binarization methods. The difference in the discrimination case proportion to texture overlap and contrast amongst classes shows that the normal class exceeds the first prize for the abnormal class. Random approach of fractal dimensions' calculations requires making no processes on all texture images (like the approach of binarization) it arbitrarily selects the boxes, thereby having less processing and variable values for fractal dimension values compared to the approach of binarization. Fractal dimension is precise in describing textures of images and accounting their properties; it obtains the highest recognition percentage compared to the rest of the used methods that have obtained a rate of identification equal to $96 \%$ and $70 \%$ on the approach of binarization and random box method frequently for all of the classes.

\section{FUTURE WORK}

In future work we can use the evolutionary computation and neural networks for training and testing the features. Improve the mechanism of determining the multifractal dimension to speed up the classification process and some statistical or structural attributes to improve the quality of segmentation for the fractal dimension.

\section{REFERENCES}

[1] M. Cimpoi, S. Maji, I. Kokkinos, and A. Vedaldi, "Deep filter banks for texture recognition, description, and segmentation," International Journal of Computer Vision, vol. 118, no. 1, pp. 65-94, 2016.

[2] J. Zhang, M. Marszałek, S. Lazebnik, and C. Schmid, "Local features and kernels for classification of texture and object categories: A comprehensive study," International Journal of Computer Vision, vol. 73, no. 2, pp. 213-238, 2007.

[3] R. K. Senapati, "Bright lesion detection in color fundus images based on texture features," Bulletin of Electrical Engineering and Informatics, vol. 5, no. 1, pp. 92-100, March 2016.

[4] F. Z. Jabr and A. A. Mohammed, "Diagnosing of some hepatic lesions from light microscope images based on morphological and texture features," Indonesian Journal of Electrical Engineering and Computer Science, vol. 18 no.2, pp. 995-1003, 2020.

[5] X. Xianghua, "A review of recent advances in surface defect detection using texture analysis techniques," ELCVIA: Electronic Letters on Computer Vision and Image Analysis, vol. 7, no.3, pp.1-22, 2008.

[6] B. B. Mandelbrot and J. A. Wheeler, "The fractal geometry of nature," American Journal of Physics, vol. 51, no. 3, pp. 1-14, 1983.

[7] C. J. Hart, "Implicit representations of rough surfaces," Computer and Graphics Forum, vol. 16, no. 2, pp. 91-99, 2008. 
[8] S. Al-Shaban, I. A. M. Al-Sadik, and M. A. Amir, "Blood cells images-based on chaos theory," Research Journal of Applied Sciences, Engineering and Technolog, vol. 1, no. 3, pp. 121-124, 2009.

[9] A. Tzalavra, K. Dalakleidi, E. I. Zacharaki, N. Tsiaparas, F. Constantinidis, N. Paragios, and K. S. Nikita, "Comparison of multi-resolution analysis patterns for texture classification of breast tumors based on DCE-MRI," International Workshop on Machine Learning in Medical Imaging, pp. 296-304, 2016.

[10] L. Guan, H. Yifeng, and K. Sun-Yuan, "Multimedia image and video processing-Second edition," Taylor \& Francis Group, CRC press, 2012

[11] K. I. Ahmed, M. H. Habaebi, and Md. R. Islam, "Smartphone aided real-time blood vein detection system," Bulletin of Electrical Engineering and Informatics, vol. 8, no. 3, pp. 1096-1107, Sept. 2019.

[12] S. N. M. Safuan, M. R. Md Tomari, W. N. W. Zakaria, M. N. H. Mohd, and N. S. Suriani, "Computer aided system for lymphoblast classification to detectacute lymphoblastic leukemia," Indonesian Journal of Electrical Engineering and Computer Science, vol. 14, no. 2, pp. 597-607, May 2019.

[13] A. S. A. Salam, M. N. M. Isa, and M. I. Ahmad, "M7 subtype leukemic cell edge detection techniques with threshold value comparison and noise filters," Indonesian Journal of Electrical Engineering and Computer Science, vol. 13, no. 3, pp. 1294-1302, March 2019.

[14] Sh. M. Rosli, M. M. Rosli, and R. Nordin, "A mapping study on blood glucose recommender system for patients with gestational diabetes mellitus," Bulletin of Electrical Engineering and Informatics, vol. 8, no. 4, pp. 1489-1495, Dec. 2019

[15] A. S. A. Salam, M. N. M. Isa, and M. I. Ahmad, "Spatial domain image enhancement techniques for acute myeloid leukemia (M1, M4, M5, M7)," Indonesian Journal of Electrical Engineering and Computer Science, vol. 14, no. 1, pp. 250-257, April 2019.

[16] J. B. Florindo and B. M. Odemir, "Texture analysis by multi-resolution fractal descriptors," Expert Systems with Applications, vol. 40, no. 10, pp. 4022-4028, 2013.

[17] R. Abiyev and I. K. Kemal, "An efficient fractal measure for image texture recognition," IEEE Fifth International Conference on Soft Computing, Computing with Words and Perceptions in System Analysis, Decision and Control, pp.1-4, 2009.

[18] R. S. Nayak and M. Jibitesh, "An improved method to estimate the fractal dimension of colour images," Perspectives in Science, vol. 8, pp. 412-416, 2016.

[19] H. Al-Sahaf, B. Xue, and M. Zhang, "A multitree genetic programming representation for automatically evolving texture image descriptors," in Asia-Pacific Conference on Simulated Evolution and Learning, pp. 499-511, 2017.

[20] H. Liu and L. Yu, "Toward integrating feature selection algorithms for classification and clustering," IEEE Transactions on Knowledge and Data Engineering, vol. 17, no. 4, pp. 491-502, 2005.

[21] Z. F. Jabr, R. H. Hussain, and S. R. Saleh, "Arrhythmia detection based on combination of freeman chain code and first order texture features," Journal of Theoretical and Applied Information Technology, vol. 97, no. 1, pp. 207$214,2019$.

[22] M. A. Shahin, M. B. Jaksa, and H. R. Maier, "Artificial neural network applications in geotechnical engineering," Australian Geomechanics, vol. 36, no. 1, pp. 49-62, 2001.

[23] G. Kylberg, "The Kylberg texture dataset v. 1.0, Centre for Image Analysis, Swedish University of Agricultural Sciences and Uppsala University, External report (Blue series) No. 35," 2011. [Online]. Available at: http://www. cb. uu. se/ gustaf/texture.

[24] T. Prakash, "Medical image processing methodology for liver tumour diagnosis," International Journal on Soft Computing (IJSC), vol. 8, no. 3/4, pp. 13-19, 2017.

[25] A. F. H. Alharan, H. K. Fatlawi, and N. S. Ali, "A cluster-based feature selection method for image texture classification," Indonesian Journal of Electrical Engineering and Computer Science, vol. 14, no. 3, pp. 1433-1442, 2019.

\section{BIOGRAPHIES OF AUTHORS}

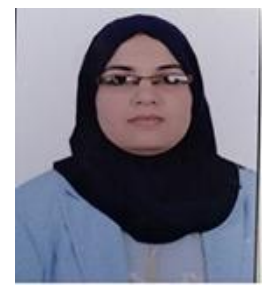

Wijdan Jaber AL-kubaisy received the B.Sc. (honors) (first class) degree in Computer Science from Al-Anbar University, Ramadi, Iraq, in 2005, and the M.Sc. degree in Computer Science from Al-Anbar University, Baghdad, Iraq, in 2017. She is currently an Assistant Lecture at the College of Computer Science and Information Technology, University of Anbar.

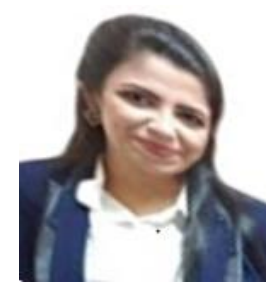

Maha Mahmood received the B.Sc. (honors) (first class) degree in Computer Science from University of Anbar, Ramadi, Iraq, in 2009, and the M.Sc. degree in Computer Science from Universityof Anbar, Ramadi, Iraq, in 2015. She is currently an Assistant Lecturer at the College of Computer Science and Information Technology, University of Anbar. She has published over 10 refereed journal and conference papers. Her current research interests include evolutionary and adaptive learning particularly in computer games, expert systems, and heuristics and meta/hyper- heuristics. She has a particular interest in neural networks. Maha Mahmood is a reviewer of four conferences and TPC member of five conferences. 